Apidologie, 1987, 18 (3), 267-278

\title{
NEUE QUALITÄTSKRITERIEN BEI HONIGUNTERSUCHUNGEN
}

\author{
S. BOGDANOV *, K. RIEDER ** und M. RÜEGG *
}

* Eidgenössische Forschungsanstalt für Milchwirtschaft, Sektion Bienen, Liebefeld-Bern, Schweiz

** Kantonales Laboratorium, Bern, Schweiz

\section{ZUSAMMENFASSUNG}

Im Rahmen einer Lebensmittelmarktkontrolle wurden 37 Honige in- und ausländischer Herkunft mit physikochemischen Methoden untersucht. Neben cingebürgerten Honigqualitätsmerkmalen wic Wassergehalt, Proteingchalt, Diastase und Saccharascaktivität, HMF-Gchalt, Leitfähigkeit und Zuckerspektrum haben wir cinige neue Qualitätskriterien wie Wasseraktivität, Honigfarbe, Absorption von Honiglösungen, sowie antibakterielle Aktivität, gemessen.

Diese neuen Qualitätskriterien wurden mit den bestehenden verglichen und ihre Bedeutung bei der Honigbcurtcilung diskuticrt.

\section{EINLEITUNG}

Die Honigbeurteilung nach dem Schweizerischen Lebensmittelbuch (Schweizerisches Lebensmittelbuch, 1967) basiert auf einigen, seit Jahren eingebürgerten Qualitätskriterien. Man beschränkt sich meistens auf die Feststellung allfälliger Wärmeschäden, indem man den HMF Gehalt und die Enzymaktivität (Diastase, Saccharase) des Honigs misst.

Will man noch den Honig über seine botanische Herkunft (Nektar- oder Honigtau) oder über allfällige Verfälschungen prüfen, so kommen dazu : Pollenanalyse, pH Bestimmung mit Säuretitration, Proteinbestimmung, sowie Bestimmung des Zuckerspektrums und des Aschengehalts. Letzteres wird in der letzten Zeit oft durch die Messung der Leitfähigkeit ersetzt. Das Ziel dieser Arbeit war, einige neue Qualitätskriterien auf ihre Anwendbarkeit bei der Honigbeurteilung zu prüfen. Dies waren die Wasseraktivität, die Honigfarbe, die Lichtabsorption von Honiglösungen, der Gehalt an Melezitose und Raffinose, sowie die antibakteriellen Eigenschaften. 


\section{MATERIAL UND METHODEN}

37 Honigproben in- und ausländischer Herkunft wurden zwecks Marktkontrolle vom Kantonschemiker in Bern erhoben.

Einige auscrwählte Proben wurden pollenanalytisch untersucht, um festzustellen, ob die Herkunftsangaben (botanisch und geographisch) stimmten.

Der Wassergehalt (refraktometrisch) und der Proteingehalt nach Lund wurden nach Lebensmittelbuch bestimmt. Für die Amylase (Siegenthaler, 1975 und Bogdanov, 1984) und Saccharase (SiegenthaLER, 1977) wurden neue Methoden, die auf Reaktionen mit künstlichen Substraten beruhen, verwendet. In der Schweiz haben sich diese Methoden bei der Honiganalyse dank ihrem geringen Zeitaufwand gegenüber den alten Lebensmittelbuchmethoden, durchgesetzt. Der HMF Gehalt wurde mit HPLC bestimmt (Iluring and Kippers, 1980). Die Nachweisgrenze ist bei 2 ppm. Die Methode lieferte bei uns gleiche Werte wie die p-Toluidin Methode nach WinkLer (WinkLer, 1955), der Vorteil liegt darin, dass auf das kanzerogene p-Toluidin verzichtet werden kann. Die Leitfähigkeit von $20 \%$-igen Honiglösungen (auf die Honigtrockensubstanz bezogen) wurde mit einem Metrohm E 365 B Konduktoskop bei $20^{\circ} \mathrm{C}$ gemessen. Die Monosaccharide Glukose und Fruktose wurden enzymatisch mit Böhringer (Mannheim) Test-Kits bestimmt. Die Zucker Melezitose und Raffinose wurden dünnschichtchromatographisch (GAUCH et al., 1979) durch Vergleich mit Standards semiquantitativ erfasst.

Für die Messung der antibakteriellen Wirkung wurden 2 Methoden verwendet (Bogdanov, 1983) : Die hitzelabilen Inhibine, die durch Wasserstoffperoxidentwicklung des Honigs wirken, wurden mit der Messung der Glukoseoxidase erfasst. Die Aktivität der hitzestabilen Inhibine wurde turbidometrisch mit Staphylococcus aureus bestimmt. Unter diesen Bedingungen hatte $\mathrm{H}_{2} \mathrm{O}_{2}$ keinen Einfluss auf das Wachstum von Staphylococcus aureus.

Die Wasseraktivität wurde nach RüEgG und Blanc (RüEgG and Blanc, 1981) bestimmt. Die Absorption von $20 \%$-igen Honiglösungen wurde in einem Perkin Elmer Doppelstrahl Spektrophotometer Model 165, bestimmt.

Die Honigfarbe wurde mit einem Munsellfarbenatlas (ZürCHer et al., 1975) bestimmt. Für diesen Zweck wurden die Honige in einem durchsichtigen $30 \mathrm{ml}$ Plastikbecher auf die $20 \mathrm{ml}$ Marke eingefüllt und gegen weissen Hintergrund der entsprechenden Farbe vom Farbatlas zugeordnet.

\section{RESULTATE}

\section{Lebensmittelrechtliche Beurteilung}

- Herkunft (geographisch, botanisch) Pollenanalyse eines «Veltlinerhonigs zeigte, dass der Honig nicht aus diesem Gebiet stammte - es kam eher eine Herkunft aus Mittel- oder Süditalien in Frage.

- Qualität : Nur eine Probe erfüllte die Qualitätskriterein des Lebensmittelbuches nicht (stark erhitzt).

- Rückstände : Es konnten weder Sulfathiazol (Nachweisgrenze 0,1 ppm) noch Fumidil (Nachweisgrenze 0,5 ppm) in den Honigen nachgewiesen werden.

\section{Wassergehalt, Wasseraktivität und Honigkristallisation}

Es ergab sich eine signifikante Korrelation zwischen Wassergehalt und Wasseraktivität des Honigs : Korrelationskoeffizient $(r)=0,784, p<0,001$. 
Die mittlere Wasseraktivität der kristallisierten Honigproben war $0,595 \pm 0,029$, die der gleichen, aber verflüssigten Honige $0,575 \pm 0,023$. Das bedeutet, dass die kristallisierten Honige durchschnittlich $1,1 \%$ mehr Wasser enthielten als die gleichen verflüssigten Proben. Bei einzelnen Proben betrug der Unterschied bis 3,2\%. 10 der 37 Honigproben waren noch flüssig, die anderen waren mehr oder weniger fest kristallisiert. $80 \%$ der flüssigen Proben hatten einen Glukosegehalt kleiner als 27,8\%/100 G Honig und einen G/W Wert (Glukosegehalt dividiert durch den Wassergehalt des Honigs) kleiner als 1,69. Bei den kristallisierten Proben hatten $85 \%$ der Proben Glukosegehalt grösser als 27,8 und $73 \%$ der Proben G/W Werte grösser als 1,69.

\section{Antibakterielle Eigenschaften (Inhibine)}

In Tab. 1 werden die antibakteriellen Eigenschaften mit anderen Qualitätskriterien der untersuchten Honigproben korreliert. Die Aktivität der hitzestabilen Inhibine korreliert signifikant nur mit der Saccharase- und Diastaseaktivität, diejenige der hitzelabilen Inhibine $\left(\mathrm{H}_{2} \mathrm{O}_{2}\right.$-Produktionskapazität) korreliert noch zusätzlich negativ mit dem HMF-Gehalt. Es wurde keine signifikante Korrelation zwischen den beiden Inhibinsorten untereinander und mit den anderen Messgrössen festgestellt. Die Aktivität der beiden Inhibine war unabhängig von der botanischen (Blüten- oder Honigtau) oder der geographischen (In- oder Ausland) Herkunft der Proben.

TAB. 1. - Antibakterielle Eigenschaften von Honig: Korrelation mit HMF-Gehalt, Saccharasezahl (SZ) und Diastasezahl (DZ).

TABL. 1. - Antibacterial properties of honey : correlation with HMF content, saccharase (SZ) and diastase number (DZ).

\begin{tabular}{l|c|c|c|c|c|c}
\hline \hline & \multicolumn{2}{|c|}{ HMF } & \multicolumn{2}{c|}{ SZ } & \multicolumn{2}{c}{ DZ } \\
\cline { 2 - 7 } & $\mathrm{r}$ & $\mathrm{p}$ & $\mathrm{r}$ & $\mathrm{p}$ & $\mathrm{r}$ & $\mathrm{p}$ \\
\cline { 2 - 7 } $\begin{array}{l}\text { Hitzestabile Inhibine } \\
\text { Heat-stable inhibines } \\
\begin{array}{l}\text { Hitzelabile Inhibine } \\
\text { Heat-labile inhibines }\end{array}\end{array}$ & -0.407 & 0.013 & 0.312 & 0.061 & 0.394 & 0.017 \\
\hline
\end{tabular}

$\mathbf{r}=$ correlation coefficient.

\section{Beurteilung von Wärmeschäden}

Aus Fig. 1 ist die Beziehung des HMF Gehalts zur Diastase- und der Saccharaseaktivität ersichtlich. Es gibt hochsignifikante negative Korrelationen zwischen den Enzymaktivitäten einerseits und dem HMF Gehalt andererseits. 

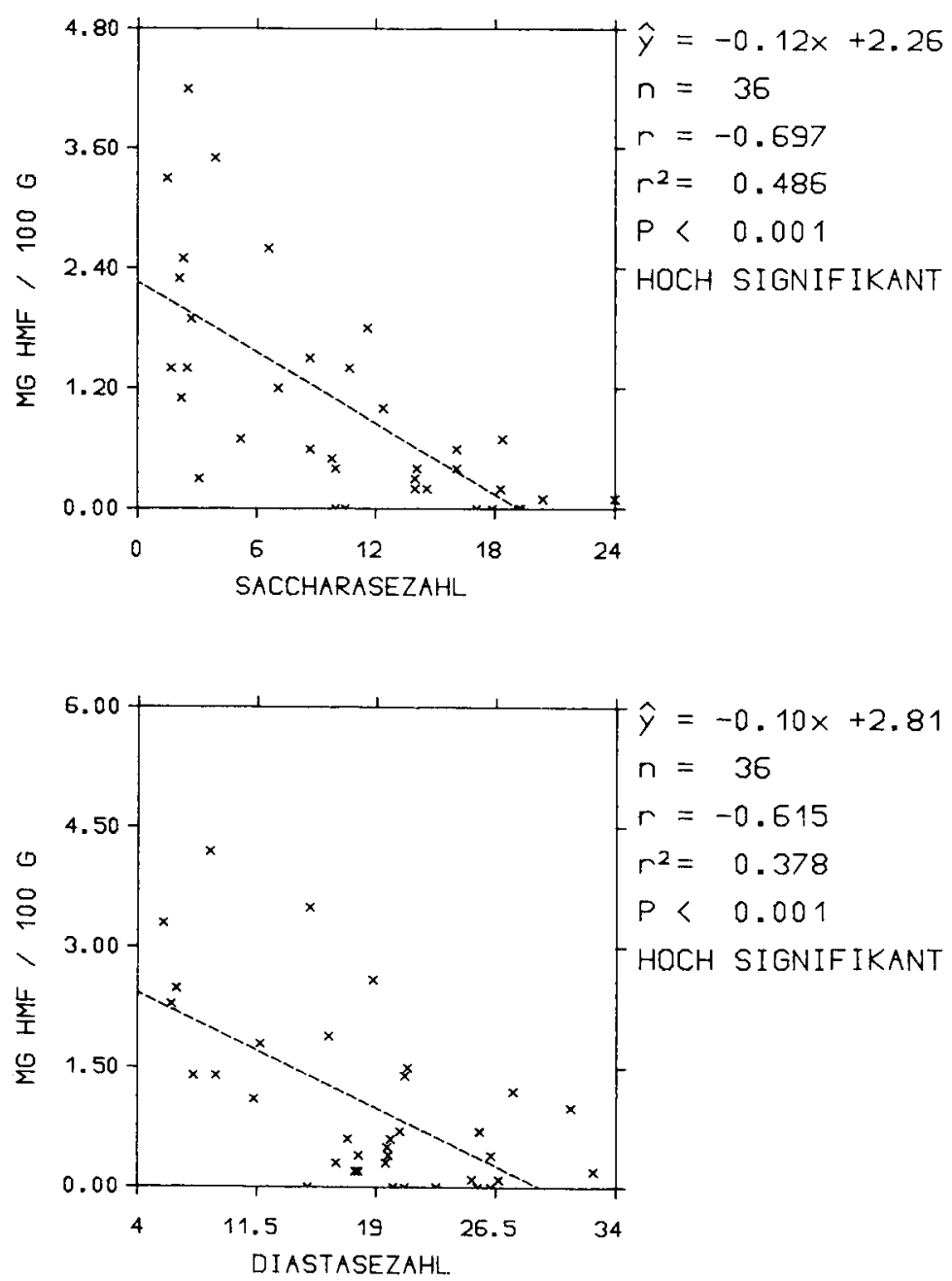

Aвв. 1. - Korrelation von HMF-Gehalt und Saccharasezahl (A) and zwischen HMF-Gehalt und Diastase-Zahl (B)

FIG. 1. - Correlation between HMF-content and Saccharase number (A) and between $H M F$-content and Diastase number (B)

Dieser Zusammenhang (statistisch definiert durch das Bestimmtheitsmass r) ist gesichert bei der Korrelation HMF/Saccharasezahl nur in $49 \%$ der Proben, bei der Korrelation HMF-Diastasezahl bei $39 \%$ der Proben. Insbesondere haben Honige mit tiefen Enzymaktivitäten sowohl tiefe wie auch hohe HMF Werte. 
Geographische Herkunft des Honigs und chemische Qualitätskriterien

In Tab. 2 sind die Werte für Wassergehalt, Diastase- und Saccharaseaktivität und HMF Gehalt für die inländischen und ausländischen Honigproben zusammengestellt. Für alle 4 Qualitätskriterien bestehen signifikante Unterschiede zwischen beiden Honiggruppen : die grössten Unterschiede sind beim Wassergehalt und HMF-Gehalt, die kleinsten in der Diastasezahl. Bezüglich diesen Qualitätsmerkmalen unterschieden sich Blüten- und Honigtauhonigen nicht signifikant voneinander.

TAB. 2. - Wassergehalt, Wasseraktivität, Diastasezahl, Saccharasezahl

und HMF-Gehalt in inländischen $(n=14)$ und ausländischen Honigen $(n=22)$.

TABL. 2. - Water content, Diastase number, Saccharase number and HMF content in honeys of Swiss $(n=14)$ and foreign origin $(n=22)$.

\begin{tabular}{l|c|c|c|c|c}
\hline \hline & $\begin{array}{c}\text { Wasser } \\
\text { water }\end{array}$ & a-W & HMF & SZ & DZ \\
\cline { 2 - 6 } & $15.7 \pm 0.7$ & $0.572 \pm 0.002$ & $0.23 \pm 0.02$ & $14.0 \pm 0.4$ & $21.4 \pm 0.3$ \\
$\begin{array}{l}\text { Inland } \\
\text { Swiss } \\
\begin{array}{l}\text { Ausland } \\
\text { Foreign }\end{array}\end{array}$ & $17.9 \pm 0.4$ & $0.607 \pm 0.001$ & $1.57 \pm 0.05$ & $8.1 \pm 0.3$ & $16.9 \pm 0.4$ \\
\hline
\end{tabular}

a-W - Wasseraktivität.

A-W - Water asctivity.

Mittelwerte \pm Standartfehler des Mittelwertes.

Mean values \pm standard error of the mean.

\section{Botanische Herkunft des Honigs (Honigtau/Nektar)}

Auf Fig. 2 sind die Absorptionsspektren im sichtbaren und nahen UV Wellenlängenbereich von einigen der untersuchten Honige wiedergegeben. Bei allen Honigen war ein Maximum bei $340 \mathrm{~nm}$ zu beobachten. Die Extinktionen bei 3 Wellenlängen $(340,400$ und $450 \mathrm{~nm})$ korrelierten sehr gut $(\mathrm{p}<0,001)$ miteinander. Wir haben uns für die Extinktion bei $340 \mathrm{~nm}$ entschieden, da dieser Wert am besten mit den anderen Kriterien für Honigherkunft (Leitfähigkeit, Monosacchariden- und Trisaccharidengehalt) korrelierte. Der Zusammenhang zwischen einigen Messgrössen und der Honigherkunft (Honigtauhonig oder Blütenhonig) ist in Tab. 3 wiedergegeben. Am besten kann man zwischen beiden Honigsorten mit Hilfe des Gehalts an Monosacchariden (Glukose und Fruktose) unterscheiden : damit konnten alle Honigtauhonige und $92 \%$ der Blütenhonige richtig zugeordnet werden. Mit dem Gehalt der Trisaccharide Melezitose und Raffinose konnten $100 \%$ der Blütenhonige und $91 \%$ der Honigtauhonige richtig zugeordnet werden. Mit dem vom Auge zugeordnete 


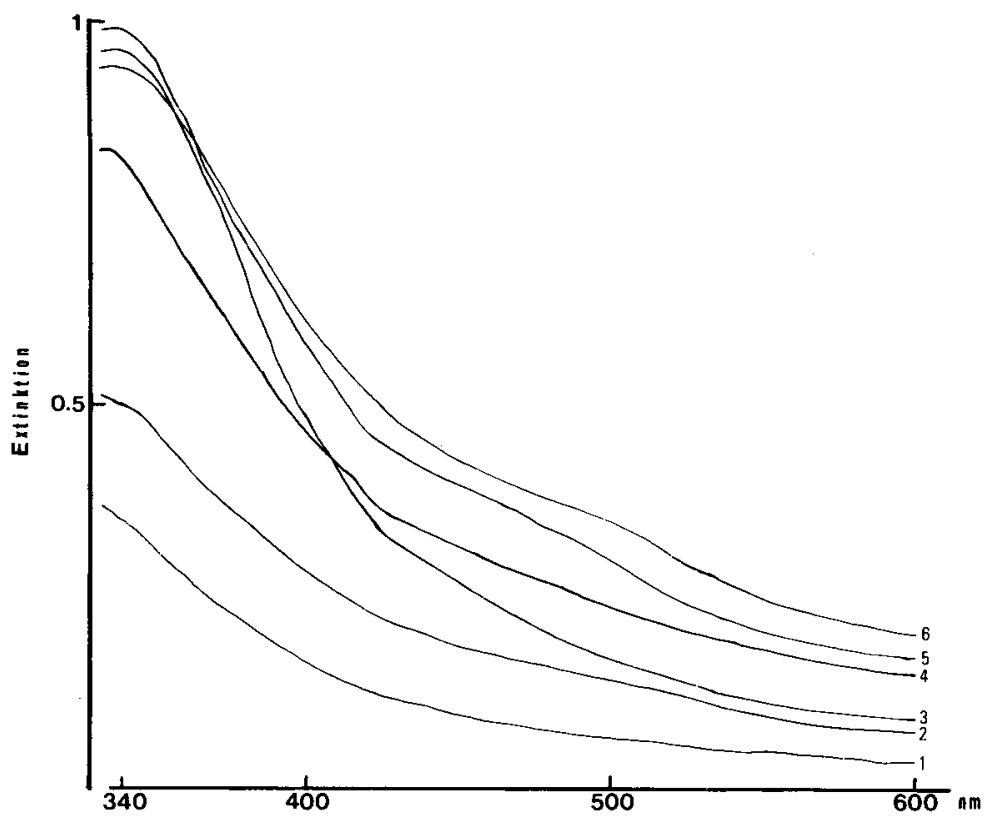

Авв. 2. - Absorptionspektren von Honiglösungen.

Proben 1, 2, 4 und 6: Blütenhonige, Probe 3 und 5: Honigtauhonige

FIG. 2. - Absorption spectra of honey solutions.

Samples 1, 2, 4 and 6 : flower honey, samples 3 and 5 : honey-dew honey

TAB. 3. - Zusammenhang zwischen Trachtherkunft (Honigtau oder Nektar) und chemischen Qualitätskriterien von Honig.

TABL. 3. - Correlation between origin (honey-dew or nectar) and chemical propertics of honey.

\begin{tabular}{l|c|c|c|c|c}
\hline \multirow{2}{*}{$\begin{array}{c}\text { Messparameter } \\
\text { Parameter }\end{array}$} & \multicolumn{2}{|c|}{$\begin{array}{c}\text { Honigtau } \\
\text { Honey-dew }\end{array}$} & \multicolumn{3}{c}{$\begin{array}{c}\text { Blüten } \\
\text { Flower }\end{array}$} \\
\cline { 2 - 6 } & $\mathrm{m} \pm \mathrm{s}$ & $\%$ & $\mathrm{~m} \pm \mathrm{s}$ & $\%$ & $\mathrm{D}$ \\
\hline $\begin{array}{l}\% \text { Monosaccharide } \\
\% \text { Monosaccharides } \\
\% \text { Trisaccharide } \\
\% \text { Trisaccharides }\end{array}$ & $58.4 \pm 2.0$ & 100 & $68.5 \pm 4.2$ & 92 & 63.5 \\
$\begin{array}{l}\text { Leitfähigkeit, mS } \\
\text { Conductivity }\end{array}$ & $4.0 \pm 2.2$ & 91 & $0.80 \pm 0.40$ & 100 & 1.7 \\
$\begin{array}{l}\text { E - 340 nm } \\
\begin{array}{l}\text { Farbhelligkeit nach Munsellatlas } \\
\text { Colour intensity after Munsell }\end{array}\end{array}$ & $1.20 \pm 0.20$ & 91 & $0.34 \pm 0.17$ & 92 & 0.78 \\
\hline
\end{tabular}

$\mathrm{m} \pm \mathrm{s}:$ Mittelwertc \pm Standardabweichung. Mean value \pm standard deviation.

D : Diskriminationswert $=$ Grenzwert, mit dem zwischen den beiden Honigklassen unterschieden wird. Discriminatory value to distinguish between flower- and honey-dew honeys.

$\%: \%$ der Honige die mit dem D Wert richtig zugeordnet wurden.

$\%$ of the honcys which were discriminated correctly with the $D$ value. 
Munsell-Helligkeitsklasse konnten die Honigtauhonige gleich gut zugeordnet werden wie mit der Bestimmung der Absorption der Honiglösungen. Bei den Blütenhonigen war die Helligkeitsklasse das etwas bessere Unterscheidungskriterium als die Absorption der Honiglösung. Die meisten Blütenhonige wurden den Helligkeitsklassen 7 bis 8,5 zugeordnet, währenddem die Honigtauhonige mehrheitlich der Helligkeitsklasse 5 zugeordnet wurden. Zwei Honige aus Kastanientracht würden mit der Leitfähigkeit, mit der Farbenhelligkeit und mit der Honigabsorption dem Honigtauhonig zugeordnet, mit dem Gehalt an Mono- und Trisacchariden jedoch dem Blütenhonig zugeordnet.

\section{DISKUSSION UND SCHLUSSFOLGERUNGEN}

\section{Wassergehalt, Wasseraktivität und Kristallisation}

Nicht der Wassergehalt, sondern die Wasseraktivität des Honigs ist für das mikrobielle Wachstum, also auch für die Honigfermentation massgebend. Währenddem es grundlegende Arbeiten über die Honigwasseraktivität gibt (RüEGG and Blanc, 1981 ; Tabouret, 1979), fehlen Arbeiten, die den Zusammenhang zwischen Honigfermentation und Wasseraktivität untersucht haben. Dies ist ein komplexes Arbeitsgebiet, spielen doch die Hefeart, die Hefekonzentration und die Hefeaktivität auch eine Rolle. Es ist bekannt, dass rasch kristallisierbare Honige wie der Rapshonig besonders fermentationsgefährdet sind. Dies könnte auf die Erhöhung der Wasseraktivität des Honigs nach der Kristallisation zurückzuführen sein. 4 der untersuchten Blütenhonige waren Rapshonige. Der Unterschied der Wasseraktivität zwischen festen und verflüssigten, gleichen Rapshonigen betrug durchschnittlich 0,017 und war nicht grösser als bei den übrigen Blütenhonigen. Ob die erhöhte Fermentationsgefahr der Rapshonige auf die höhere Wasseraktivität als bei den übrigen Blütenhonigen zurückzuführen ist, sollte auf Grund von umfassenderen Untersuchungen entschieden werden.

Nach WhITE et al. (1962) soll die Kristallisationstendenz des Honigs am besten mit dem Verhältnis Glukose/Wasser (G/W) voraussagbar sein. Danach sollten Honige mit Glukosegehalt < als $28 \mathrm{~g} / 100 \mathrm{~g}$ Honig und G/W-Werte < als 1,7 flüssig bleiben, währenddem solche mit grösseren Werten mehr oder weniger rasch kristallisieren. Wir fanden aber, dass bei den kristalliserten Honigen der Glukosegehalt etwas bessere Voraussagekraft als das G/W hatte. Der in dieser Arbeit bestimmte Kristallisationsdiskriminationswert von 27,8\% Glukose, sowie ein Verhältnis von Glukose/Wasser von 1,69 waren mit den Werten von White praktisch identisch. HADORN und ZürCHER (1974) fanden jedoch, dass fast $50 \%$ der Honige mit $\mathrm{G} / \mathrm{W}>$ als 1,7 noch flüssig waren. 


\section{Antibakterielle Eigenschaften}

Bogdanov (1983) konnte auf Grund von mikrobiologischen und chemischen Tests 2 Inhibinklassen in Honig nachweisen. Die Hitze- und lichtempfindlichen Inhibine haben bekannlich ihren Ursprung im Honig Wasserstoffperoxid, dessen Entwicklung durch die Glukose Oxidase und die Katalase gesteuert wird (Bogdanov, 1983 und dort weitere Literatur). Die übrige antibakterielle Aktivität des Honigs beruht auf hitze- und lichtunempfindlichen Inhibinen. Die Saccharase- und Diastase Aktivitäten korrelierten besser mit den hitzelabilen, als mit den hitzestabilen Inhibinen. Dies könnte auf die verschiedene Beschaffenheit beider Inhibinsorten zurückzuführen sein. Die Glukoseoxidase (hitzelabile Inhibine) sowie die Saccharase und die Diastase stammen von der Biene und werden bei der Honigbearbeitung von der Biene zugesetzt - damit kann die gute Korrelation dieser Enzymaktivitäten erklärt werden. Der Befund, dass nur die Aktivität der hitzeempfindlichen Inhibine negativ mit dem HMF Gehalt korreliert, kann auf die relative Unempfindlichkeit der hitzestabilen Inhibine gegen Hitze und Lagerung (Bogdanov, 1983) zurückführt werden. Die Tatsache, dass die hitzestabilen Inhibine signifikant mit der Saccharase- und Diastaseaktivität korrelieren, könnte bedeuten, dass wenigstens ein Teil der hitzestabilen Inhibine von der Biene stammen. Damit sind wohl die Befunde zu erklären, dass Blüten- und Honigtauhonige sich nicht in der Aktivität der hitzestabilen Inhibine unterscheiden (diese Untersuchung), sowie dass auch Zuckerfütterungshonige über beträchtliche Aktivität der hitzestabilen Inhibine verfügt (S. Bogdanov, unveröffentlichte Beobachtungen). Die von uns im Honig nachgewiesenen und antimikrobiell wirksamen Honigflavonoide können auf Grund ihrer geringen Konzentration nur unwesentlich zur Aktivität der hitzestabilen Inhibine beitragen (Bogdanov, 1983). Die Existenz vom antibakteriell wirksamem und relativ hitzeresistentem Lysozym (MoHRIG und Messer, 1968) konnte von uns nicht bestätigt werden (Bogdanov, 1983). Die chemische Natur dieser Inhibine bleibt vorläufig unbekannt.

\section{Geographische Herkunft der Proben und chemische Qualitätskriterien}

Die Honigbeurteilung in der Schweiz, aber auch in anderen europäischen Ländern beschränkt sich meistens auf die Bestimmung der Wärmeschäden des Honigs, die mit Hilfe der Messung von Diastase- und Saccharaseaktivität und HMF Gehalt beurteilt wird (Hadorn et al., 1962, Duisberg und Hadorn, 1966). Diese Gehaltszahlen werden als Mass für die Naturbelassenheit des Honigs genommen (Schweizerisches Lebensmittelbuch, 1967). Der Unterschied in diesen Gehaltszahlen hat einige Länder, wie z.B. die BRD dazugeführt, für den inländischen "Qualitätshonig » strengere Normen für HMF und Saccharaseaktivität als für den übrigen Handelshonig, einzuführen. Der Unterschied bei den Enzymaktivitäten und im HMF Gehalt der in- und ausländischen Proben 
(Tab. 2) kann mit dem Alter und/oder mit der verschiedenen Wärmebehandlung von beiden Honiggruppen erklärt werden : der inländische Honig gelangt meistens frisch auf den Markt, der ausländische Honige hat hingegen einen langen Transportweg hinter sich und wird dazu noch meistens zwecks Abfüllung mit mehr oder weniger Wärme behandelt. Der Unterschied im Wassergehalt könnte mit der verschiedenen Produktionsweise der beiden Honiggruppen erklärt werden : in der Schweiz gibt es keine "Honigindustrie", bei der auf die Reifung des Honigs genügend geachtet wird. Der ausländische Honig in der Schweiz kommt meistens von Ländern mit einer grossen Honigproduktion, wo unweigerlich mehr unreifer Honig produziert wird.

\section{Botanische Herkunft des Honigs}

Der Konsument unterscheidet zwischen Honigtau- und Blütenhonig auf Grund der Honigfarbe : der helle Honig wird als Blütenhonig, der dunkle als Waldhonig taxiert. Diese Regel hat Ausnahmen : der Kastanienhonig und der Heidehonig sind dunkle Blütenhonige. Auch gibt es manchmal helle Honigtauhonige, besonders bei Spättrachten. Im Honighandel wird die Honigfarbe durch Farbenvergleich ermittelt. In Europa ist das Pfund System am gebräuchlichsten. Ausserdem haben ZürCHER et al. (1975) einen Honigfarbenatlas mit Munsellfarbenplättchen zusammengestellt, mit dessen Hilfe die Honigfarbe einfach bestimmt werden kann. Dabei bestimmt man die Farbe, sowie ihre Helligkeit und Tiefe. Die Helligkeit hat Werte von 2 (= sehr dunkel) bis 9 (= sehr hell). In dieser Untersuchung erwies sich die Farbenzuordnung mit dem Munsellfarbenatlas ebenbürtig oder sogar besser als die quantitative Messung der Absorption der Honiglösungen. Die Farbenbestimmung mit dem Farbenatlas hat dazu den Vorteil, dass der Honig mit den "Augen des Konsumenten » beurteilt wird.

Die Leitfähigkeit hat sich als ein leicht messbares Qualitätskriterium für Honigherkunft erwiesen (VorwoHL, 1964). Der Grenzwert für die Unterscheidung zwischen Honigtau- und Blütenhonigen wurde auf $0,78 \mathrm{mS} / \mathrm{cm}$ bestimmt und stimmt gut überein mit dem in der Praxis (TALPAY, 1985) angenommen Wert von $0,8 \mathrm{mS} / \mathrm{cm}$.

Die Bestimmung der reduzierenden Zucker wird heute durch spezifischere. Zuckerbestimmungsmethoden ersetzt. Hat man zur Zuckerbestimmung keine teueren Apparateneinrichtungen (HPLC oder GC), so könnte das Zuckerspektrum auch semiquantitativ mit Dünnschichtchromatographie bestimmt werden - so wie wir diese bei der Bestimmung der Mono- und Trisaccharide anwendeten. Es ist bekannt, dass Melezitose und Raffinose nur im Honigtau vorkommen, nicht aber im Nektar (Maurizio, 1975). Wir fanden, dass mit der Messung der Monosaccharide (Glukose und Fruktose) und der Trisaccharide 
(Melezitose und Raffinose) am besten zwischen Blüten- und Honigtauhonigen unterschieden werden kann.

Ein Problem stellt die Bestimmung der Herkunft des Kastanienhonigs dar. Theoretisch ist sowohl eine Herkunft aus Nektar, wie auch aus Honigtau möglich. Nach den bisher gebrauchten chemischen Kriterien würde man ihn als Honigtauhonig einstufen. 2 Kastanienhonige dieser Untersuchung wurden aber nach ihrem Gehalt an Mono- und Trisaccharide als Blütenhonige eingestuft. Eine definitive Zuordnung des Kastanienhonigs wäre erst möglich, wenn man das Zuckerspektrum des Kastanienhonigtaus bekannt ist.

Wir stellen uns eine schnelle Beurteilung der Honigherkunft (Blüten oder Honigtau) mit der Bestimmung der Honigfarbe, pH und der Leitfähigkeit als eine in der Routine gangbare Methode vor. Die Bestimmung des Zuckerspektrums könnte man eher bei der Beurteilung der spezifischen Tracht des Honigs beiziehen.

Eingegangen im August 1986. Angenommen im April 1987.

\section{DANK}

Wir danken Frau Dr. A. MAUrizıo für die Durchführung der Pollenanalyse von einigen auserwählten Proben.

\section{SUMMARY}

NEW CRITERIA FOR HONEY QUALITY

37 honeys of swiss and foreign origin were analyzed in the frame of routine food control. Besides trace insecticide analysis, the following parameters were determined: water content, water activity, colour intensity after Munsell, light absorption of honey solution, protein content after Lund, electric conductance, sugar content, HMF content, Diastase- and Saccharase activities, hydrogenperoxide production capacity (heat-labile Inhibines), and the activity of the heat- stable inhibines.

No detectable amounts of fumidile und sulfathiazol could be detected and all but one fulfilled the quality requirements required by the Swiss food legislation.

The average water activity of the crystallized honeys was measured to be 0.595 , that of the liquid or the liquified honeys 0.575 and was significantly correlated to the honey water content.

Both the glucose content and the glucose/water content ratio $(\mathrm{g} / \mathrm{w})$ could be used as prediction criterion for honey crystallization. The hydrogenperoxide accumulation activity (heat-labile inhibine) and the activity of the heat-stable inhibines were significantly correlated to the saccharase- and diastase activities. The hydrogenperoxide accumulation was negatively correlated to the HMF content of honey. There was no correlation between both sorts of inhibines and the botanical (honey-dew or floral) and the geographical (Swiss or foreign) honey origin. Also, both inhibine activities did not correlate with each other and also with the other physicol-chemical parameters. 
Swiss honeys had significantly less water, lower water activity, higher activities of saccharase and diastase and a lower content of HMF than the foreign honeys.

Honey-dew and flower honeys could be best distinguished with their content in mono- and trisaccharides, followed by electric conductance, colour intensity after the Munsell atlas and the light absorption of honey solutions.

\section{RÉSUMÉ \\ NOUVEAUX CRITĖRES DE QUALITÉ DANS L'ÉTUDE DES MIELS}

37 miels indigènes et étrangers ont été analysés dans le cadre d'un contrôle des denrées alimentaires. En plus des analyses de résidus, les paramètres suivants ont été déterminés : teneur en eau, activité de l'eau, conductibilité, intensité de la couleur du miel selon Munsell, absorption de la lumière par les solutions de miel, teneur en protéines selon Lund, teneur en sucre, teneur en HMF, activité de la diastase et de la saccharase, formation d'eau oxygénée (inhibine thermolabile), activité des inhibines thermostables.

Aucun résidu de Fumidil $(\leqslant 0,5 \mathrm{ppm})$ ni de Sulfathiazol $(\leqslant 0,1 \mathrm{ppm})$ n'a été trouvé. Un seul miel n'a pas rempli les exigences légales.

L'activité moyenne de l'eau des miels cristallisés a atteint 0,595 , celle des mêmes échantillons liquéfiés $-0,575$. Ces deux valeurs ont une corrélation significative avec la teneur en eau.

Tant la teneur en glucose que le rapport teneur en glucose/teneur en eau ont pu servir de critères d'appréciation pour la cristallisation du miel.

Nous n'avons pas trouvé de corrélation entre l'activité des inhibines thermolabiles (formation d'eau oxygénée) et celle des inhibines thermostables; elles ne dépendent pas de la provenance botanique (fleurs ou miellat) ni géographique (indigène ou étrangère) des échantillons. L'activité des deux types d'inhibines présente une corrélation significative avec celle de la diastase et de la saccharase, la formation d'eau oxygénée a produit une corrélation négative avec la teneur en HMF. Aucune corrélation signicative n'existe entre les autres paramètres et ces deux inhibines.

Les miels suisses contiennent moins d'eau, présentent une activité de l'eau plus faible, des activités de diastase et de saccharase plus élevées et ont des teneurs plus faibles en HMF que les miels étrangers, et ceci de façon significative.

La différenciation entre les miels de fleurs et ceux de miellat s'effectue le plus facilement en considérant la teneur en monosaccharides (fructose et glucose) et en trisaccharides (mélézitose et raffinose) et un peu moins bien par la détermination de la conductibilité, de l'intensité de la couleur selon Munsell ou de l'absorption de la lumière par la solution de miel.

\section{LITERATUR}

Bogdanov S., 1983. - Characterisation of antimicrobial substances in honey. Lebensm. Wiss. Technol., 17, 74-76.

Bogdanov S., 1984. - Honigdiastase : Gegenüberstellung verschiedener Bestimmungsmethode. Mitt. Gebiete lebensm. Hyg., 75, 314-220.

Duisberg H., Hadorn H., 1966, Welche Anforderungen sind an Handelshonigen zu stellen. Mitt. Gebiete Lebensm. Hyg., 57, 386-407. 
Gauch R., Leuenberger U., Baumgartner E., 1979. - Quantitative determination of mono-, di- and trisaccharides. J. Chromatogr., 174, 195-200.

Hadorn H., Zürcher, DofvelaAr F., 1962. - Ueber Wärme- und Lagerschädigungen von Bienenhonig. Mitt. Gebiete Lebens. Hyg., 53, 191-229.

HADORN H., ZürCHER K., 1974. - Zuckerspektrum und Kristallisationstendenz von Honigen. Mitt. Gebiete Lebensm. Hyg., 65, 407-420.

ILURING H., KupPERS F., 1980, - HPLC of Furfural and Hydroxymethylfurfural in Spirits and Honey. $J$. Assoc. Off. Anal. Chem., 63, 1215-1218.

Maurizio A., 1975. - How bees make honey. In : E. Crane (ed.), Honey, a comprehensive survey, London, Heinemann, 77-105.

Mohrig W., Messner B., 1968. - Lysozym als antibakterielles Agens im Bienenhonig und Bienengift. Acta. Biol. med. germ., 21, 85-95.

RÜEgG M., Blanc B., 1981. - The water activity of honey and related sugar solutions. Lebensm. Wiss. Technol., 14, 1-6.

Siegenthaler U., 1975. - Honigdiastase : Bestimmung der Amylase in Bienenhonig mit einem handelsüblichen, farbmarkierten Substrat. Mitt. Gebiete Lebensm. Hyg., 66, 393-399.

Siegenthaler U., 1977. - Eine einfache und rasche Methode zur Bestimmung der Glucoseoxidase im Honig. Mitt. Gebiete Lebensm. Hyg., 68, 251-258.

Schweizerisches Lebensmittelbuch, 5. Auflage, 2. Band, Kapitel 23 A, Abschnitt 09, Honig und Kunsthonig, Bern, 1967.

Tabouret T., 1979. - Rôle de l'activité de l'eau dans la cristallisation du miel. Apidologie, 10, 341-358.

Talpay B., 1985. - Spezifikationen für Trachthonige. Dtsch. Lebensm. Rundsch., 81, 148-151.

Vorwohl G., 1964. - Die Beziehung zwischen der elektrischen Leitfähigkeit der Honige und ihre trachtmässige Herkunft. Ann. Abeille, 7, 301-309.

WiNKLER O., 1955. - Beitrag zum Nachweis und zur Bestimmung von Oxymethylfurfural in Honig und Kunsthonig. Z. Lebensm. Unters. Forsch., 102, 161-167.

White J., Riethof M., Subers M., Kushnir I., 1962. - Composition of american honeys. Techn. Bull. 1261, Washington, DC, $24 \mathrm{p}$.

Zürcher K., Maurizio A., Hadorn H., 1975. - Untersuchungen an Handelshonigen mit spezieller Berücksichtigung des Zuckerspektrums. Apidologie, 6, 59-90. 